\title{
The Graph Drawing Server
}

\author{
Stina Bridgeman ${ }^{1}$ and Roberto Tamassia ${ }^{2}$ \\ 1 Department of Computer Science, Colgate University, Hamilton, NY 13346 USA \\ stina@cs.colgate.edu \\ 2 Center for Geometric Computing, Brown University, Providence, RI 02912 USA \\ rt@cs. brown . edu
}

\section{Short Description}

There are many obstacles in the way of someone wishing to make use of existing graph drawing technology - software installation and data conversion can be time-consuming and may be prohibitively difficult for the casual or novice user, and software may be limited to a particular platform or provided interface. The Graph Drawing Server (GDS) 2] seeks to remove many of these obstacles by providing a graph drawing and translation service with an easy-to-use webbased interface 1 . A user needs only a commonly-available web browser to access a variety of algorithms, without having to install any additional software or do any format translations (once the data is in one of many supported formats).

GDS has received over 62,000 requests from 43 countries since June 1996.

\section{Areas of Application}

Potential uses of GDS include:

- drawing user-supplied graphs,

- translating between graph descriptions in different formats,

- performing experimental comparisons of graph drawing algorithms,

- testing and developing new graph drawing algorithms,

- demonstrating graph drawing algorithms in educational settings,

- creating a database of real-life user graphs for experimental studies, and

- rapid prototyping of software containing a graph drawing component.

\section{Layout Algorithms and Layout Features}

The algorithms available through GDS come from a variety of sources - the Graph Drawing Server does not replace existing graph drawing packages, but rather brings them to a wider audience.

\footnotetext{
* Research supported in part by the NSF under grants CCR-9732327 and CDA9703080, and by the U.S. Army Research Office under grant DAAH04-96-1-0013. Work done while the first author was at Brown University.

1 http://loki.cs.brown.edu/geomNet/gds/

P. Mutzel, M. Jünger, and S. Leipert (Eds.): GD 2001, LNCS 2265, pp. 448-450, 2002.

(C) Springer-Verlag Berlin Heidelberg 2002
} 
GDS currently supports several general-purpose algorithms for orthogonal, hierarchical, and force-directed drawings, plus specialized algorithms for binary trees and series-parallel digraphs. New algorithms can be easily incorporated into the service. Output can be generated in a variety of formats including GIF and Postscript, so that drawings can easily be included in papers and presentations.

\section{Architecture}

The Graph Drawing Server is a component of GeomNet [1], a distributed geometric computing system providing access to computational geometry and graph drawing algorithms over the Internet. It utilizes the web services framework, where users connect to services running on remote machines rather than installing software packages locally.

The GDS system consists of a network of individual servers, each supporting some subset of the available algorithms. Users can send requests to any server, and that server will coordinate the others as needed to complete the request.

Individual GDS servers have a layered object-oriented architecture which facilitates adding new applications and new functionality. Current work involves adding support for enhanced security and for "cooperative computing", where client and server can negotiate the best distribution of computation in order to satisfy the request as quickly as possible.

\subsection{Programming Language}

The server component of the Graph Drawing Server is implemented in Java. Individual algorithms, translation filters, and interfaces may be implemented in any language (e.g. C, C++, Java, perl, and, for interfaces, HTML).

\subsection{Operating System}

GDS servers will run on any operating system for which a Java virtual machine exists; individual algorithms, translation filters, and interfaces may be limited to certain platforms because of language or environment requirements.

\section{Interfaces}

Users interact with GDS by sending a request consisting of the graph to be drawn, the algorithm to be run and values for its parameters, the format of the input, and the desired format for the output. Requests can be sent using a graph editor applet or a set of forms-based web pages, allowing casual or novice users to make use of the service immediately. For more advanced applications, user programs can make socket or HTTP connections directly to the service. This allows drawing algorithms to be used by any program, regardless of the platform and language of program and algorithm. 
The authors would like to thank Gill Barequet, Christian Duncan, Ashim Garg, and Michael Goodrich for their contributions to GDS and GeomNet, and Rob Mason for the creation of the graph editor applet.

\section{References}

1. G. Barequet, S. Bridgeman, C. Duncan, M. Goodrich, and R. Tamassia. GeomNet: Geometric computing over the Internet. IEEE Internet Computing, 3(2):21-29, 1999.

2. S. Bridgeman, A. Garg, and R. Tamassia. A graph drawing and translation service on the WWW. Internat. J. Comput. Geom. Appl., 9(4/5):419-446, 1999. 